\title{
Data-driven Learning to Predict WAN Network Traffic
}

\author{
Nandini Krishnaswamy \\ Lawrence Berkeley National Lab \\ Berkeley, CA, USA \\ nandinik@berkeley.edu \\ Kunal Singh \\ EECS, UC Berkeley \\ Berkeley, CA, USA \\ kunalsingh@berkeley.edu
}

\author{
Mariam Kiran \\ Lawrence Berkeley National Lab \\ Berkeley, CA, USA \\ mkiran@es.net \\ Bashir Mohammed \\ Lawrence Berkeley National Lab \\ Berkeley, CA, USA \\ bmohammed@lbl.gov
}

\begin{abstract}
In this paper, we explore both statistical and deep learning approaches for multi-step predictions in WAN traffic traces. Estimating future traffic can help improve link usage and optimize bandwidth utilization. In this paper, we study real network traces from a real WAN research network. We use Fourier analysis to present variation among the traffic traces, extracting daily and weekly peak frequencies per trace. We also develop statistical time-series methods, ARIMA and Holt-Winters, and three LSTM-based approaches with various neural network architectures (Simple, Stacked and S2S LSTM), to forecast and compare the accuracies between them.

With efforts to find a data-driven learning solution, we find that deep learning approaches can learn traffic patterns and provide more accurate predictions than ARIMA and Holt-Winters. Our results show that predictions are improved at an average of $70 \%$ or more. We further discuss the challenges of building these, their deployment and how these can help improve network utilization for future planning problems.
\end{abstract}

\section{KEYWORDS}

intent-based networking; natural language processing; smart instrumentation

\section{ACM Reference Format:}

Nandini Krishnaswamy, Mariam Kiran, Kunal Singh, and Bashir Mohammed. 2020. Data-driven Learning to Predict WAN Network Traffic. In 3rd International Workshop on Systems and Network Telemetry and Analytics (SNTA '20), June 23, 2020, Stockholm, Sweden. ACM, New York, NY, USA, 8 pages. https://doi.org/10.1145/3391812.3396268

\section{INTRODUCTION}

Wide area network (WAN) service providers are witnessing relentless traffic growth, making it a challenging task for network operators to balance network performance and capacity planning.

Permission to make digital or hard copies of all or part of this work for personal or classroom use is granted without fee provided that copies are not made or distributed for profit or commercial advantage and that copies bear this notice and the full citation on the first page. Copyrights for components of this work owned by others than ACM must be honored. Abstracting with credit is permitted. To copy otherwise, or republish, to post on servers or to redistribute to lists, requires prior specific permission and/or a fee. Request permissions from permissions@acm.org.

SNTA '20, June 23, 2020, Stockholm, Sweden

(c) 2020 Association for Computing Machinery.

ACM ISBN 978-1-4503-7980-9/20/06 . \$ \$15.00

https://doi.org/10.1145/3391812.3396268
Current trends show a surge of video streaming and mobile applications, bringing exponential traffic growth to WAN and Internet backbones [5]. This presents a need to understand how one may optimize link bandwidth and manage applications in cases of congestion. Maintaining optimum WAN bandwidth is expensive and failure can lead to packet loss due to congestion. To prevent these situations, network operators usually provision available bandwidth to $40 \%$ utilization [11] to account for sudden traffic surges during transfers. Network operators routinely make active decisions to optimize available link bandwidth, by performing traffic engineering or network reconfiguration. However, these are expensive engineering decisions and requires between 8-24 hours [1].

Capping utilization is another way in which congestion can be prevented. However, as demands grows, this becomes an unscalable solution - network link paths usually range from $10 \mathrm{~GB} / \mathrm{s}$ to $400 \mathrm{~GB} / \mathrm{s}$ in large WANs [14]. Network planning is always looking for efficient ways to manage.

In this paper, we tackle network traffic forecasting as a timeseries prediction problem to predict the next-24-hours of used bandwidth. We focus our study on comparing traditional statistical methods, such as ARIMA and Holt-Winters, along with three design variants of LSTM-based deep learning architectures, to determine a general approach that can work with any traffic pattern.

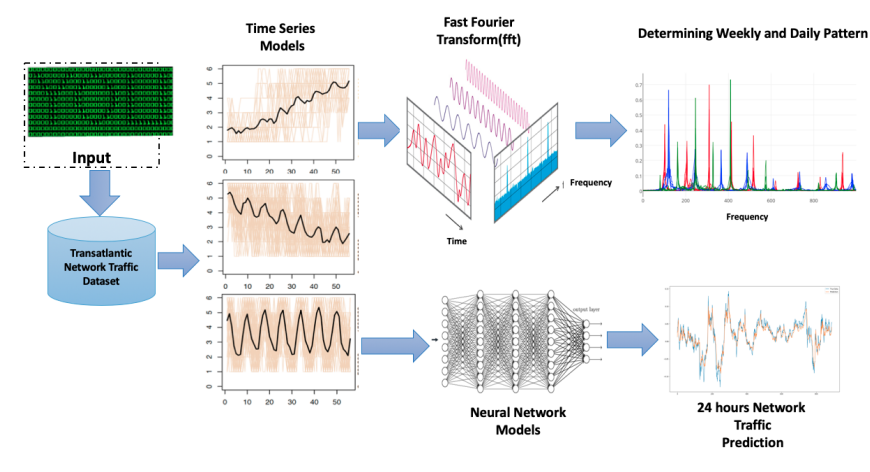

Figure 1: Studying network traffic as a time-series problem.

While applying machine learning (statistical and deep learning approaches) in network traffic prediction is not new [19], most studies only demonstrate predictions between a few seconds to 
minutes. Our work is important as it, for the first time, to the best of our knowledge, tackles hourly 24 hours predictions. Predicting traffic surges multiple hours ahead can solve many problems, such as preventing potential bottlenecks and improve network utilization. Additionally, our work tackles the problem of data-driven learning for the traffic traces themselves.

WAN and Internet backbones are an abundant and rich source of user behaviors, time zones, active users and range of file transfers that occur. Learning good time-based behavior is essential for accurate prediction tasks. Our work tackles three major contributions,

- To address data-driven learning, we develop three unsupervised learning neural network architectures - Simple LSTM, Stacked LSTM, and Sequence-to-sequence (S2S) LSTM, comparing them to statistical methods ARIMA and Holt-Winters. We evaluate these on real traffic traces from WAN research network.

- To present variability in our datasets, we conduct a detailed seasonality analysis using Fast Fourier transformation, mapping network traffic traces into a frequency domain. This helps in isolating strong frequencies and determines the daily and weekly variability.

- We discuss the computational challenges of deep learning approaches and provide results why these are powerful approaches for network research.

\subsection{Motivation: Network Traffic Forecasting Challenge}

Network traffic is extremely noisy, behaving pseudo-randomly with sudden spikes and downtimes in bandwidth. This is considered normal, as they show sudden file transfers. Developing a prediction model for these random behaviors is a formidable challenge. Further challenges include,

- Missing data: We collected real network traces from router interfaces across multiple network links, at 30-second intervals. Our analysis showed that interfaces sometimes miss logging traffic and subsequently populate the data with null values.

- Multiple hour forecasts: Predicting multiple time-steps is a difficult modeling problem. Most time-series algorithms are designed to predict one time-step ahead, and predicting multiple steps results in an error build-up that is amplified as prediction goes deeper into future.

- Unsupervised feature learning: Network traffic depends on how users or applications interact with the network and this differs based on the source, destination and link used. It can be hard to deduce and label these patterns by just monitoring traffic data. Trends on a particular day do not necessarily correlate to the next day, and seemingly arbitrary spikes of traffic need not be confined to particular time periods. Classical time-series methods like ARIMA and Holt-winters require global parameters to be set in advance based on seasonality patterns in the data. This makes them less scalable to many different traffic scenarios. Working in large WAN and Internet environments, we need to have unsupervised machine learning to can learn hidden patterns from data.
Table 1: Sample of features $\left(X_{1}, X_{2}, X_{3}, X_{4}\right)$ in SNMP. Includes start and end time, protocols, interfaces.

\begin{tabular}{llllll}
\hline $\mathrm{S} / \mathrm{N}$ & $X_{1}$ & $X_{2}$ & $X_{3}$ & $X_{4}$ & INTERFACE \\
\hline 1 & IP & TCP & ICMP & TCP & LINK1 \\
2 & IP & ICMP & TCP & UDP & LINK1 \\
$\cdot$ & $\cdot$ & $\cdot$ & $\cdot$ & $\cdot$ & $\cdot$ \\
\hline
\end{tabular}

\section{DATA SETS}

Network operators monitor link capacity and traffic by collecting and monitoring Simple Network Management Protocol (SNMP) statistics ${ }^{1}$. Along with giving information on CPU and memory utilization, SNMP collects real-time (per second) time-stamped data for traffic moving in both directions across the network link. Traffic is recorded in GBs at 30-second-intervals.

Even though other data sources on network traffic measurement protocol exist [1], SNMP collects both device and application level data by passively monitoring. Table 1 shows a sample of data features collected by SNMP.

In this study, we use real network traces from a global research WAN network (e.g. www.es.net). The traffic traces collect gigabytes (GBs) moving in each direction recorded at router interfaces with 30 -sec intervals. We aggregate this data to 1-hour intervals and model them as discrete elements. Aggregating traffic compounds the burst patterns and does not affect the accuracy of the predictions as confirmed by [7]. Additionally, aggregating the data also allows us to reduce the prediction model complexity, allowing faster training and predictions. The data sets used are 4 transatlantic twoway traffic traces collected from a global research WAN network, connecting labs in U.S. and Europe. This data is 3-months (October 2018 to January 2018) of traffic, amounting to 8 traffic traces in total. A snapshot of this data is shown in Figure 2. The transatlantic links display longer TCP return times and multi-time zone US-European connections. Our links are

(1) Amsterdam-Boston Inbound and Outbound (Amst-Bost-In, Amst-Bost-Out).

(2) Americas (Aofa)-London Inbound and Outbound (Aofa-LondIn, Aofa-Lond-Out).

(3) Washington-Cern Inbound and Outbound (Wash-Cern-In, Wash-Cern-Out).

(4) London-New York Inbound and Outbound (Lond-NY-In, LondNY-Out).

\section{STUDYING SEASONALITY USING FOURIER TRANSFORMATION}

We use FFT to extract frequency patterns across the traffic traces, to validate the variability among the traces used in this study (Figure 1). FFT extracts symmetry frequencies for signals, as finite sum of sine waves. A variation of discrete Fourier transformation equation (DFT), helps extract positive frequencies in the data set.

\footnotetext{
${ }^{1}$ Various network monitoring tools collect packet and performance data across multiple layers of a WAN, such as sflow, netflow and SNMP. In this study, we only focus on SNMP data.
} 


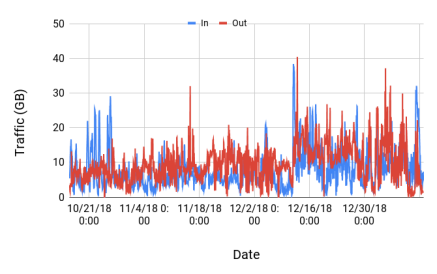

(a) Amsterdam-Boston Link.

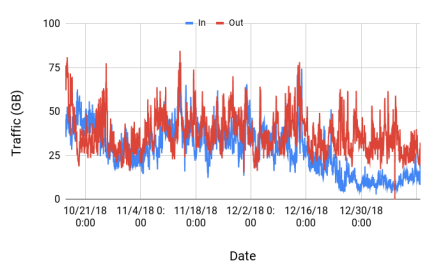

(b) Aofa-London Link.

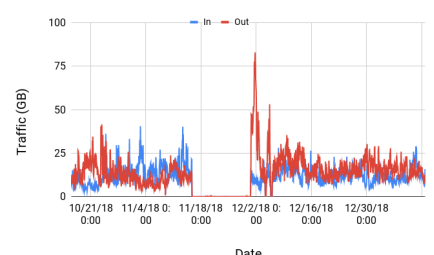

(c) Washington-Cern Link.

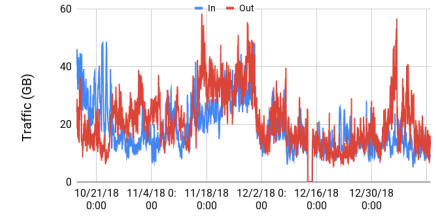

Date

(d) London-New York Link.

Figure 2: Actual traffic on transatlantic links over 3 months.

FFT can estimate the power spectra in a given signal, converting the network traffic into a time-frequency domain. It mathematically defines it into a series of sinusoidal functions where it is easier to detect seasonality. Figure 2 shows that it is difficult to extract the periodic fluctuations by just monitoring the data, as no periodic fluctuations are noticeable. FFT works by computing the DFT, transforming data from time domain to a frequency domain, shown by function transform $f$ denoted with $\hat{f}$,

$$
\hat{f}(\xi)=\int_{-\infty}^{\infty} f(x) e^{-2 \pi i x \xi} d x
$$

where $x$ represents time and $\xi$ represents the transformed frequency. In our analysis, we create a filter that eliminates low frequencies but retains high frequencies. This is because we want to analyze the daily and weekly peak frequencies and its effect on the overall network traffic data. We specify a box-shaped frequency response with cutoff frequency $\omega_{j}$ :

$$
f(\omega)= \begin{cases}1 & |\omega| \leq \omega_{j} \\ 0 & |\omega|>\omega_{j} .\end{cases}
$$

To get the power spectrum of the frequencies, we inverse the FT frequency integrating $f(\omega)$ w.r.t $\omega$,

$$
f(x)=\frac{1}{2 \pi} \int_{-\omega_{j}}^{\omega_{j}} e^{i \omega x} d \omega
$$

Substituting gives,

$$
=\left.\frac{1}{2 \pi} \frac{e^{i \omega x}}{i x}\right|_{-\omega} ^{\omega}
$$

where $\omega=\omega_{j}$. Since, $\sin \theta=\frac{e^{i \theta}-e^{-i \theta}}{2 i}$, the final equation,

$$
f(x)=\frac{w}{\pi} \operatorname{sinc}\left[\frac{w}{\pi} x\right]
$$

where $\operatorname{sinc}(x)=\sin (\pi x) /(\pi x)$. For equally spaced samples, the cutoff frequency is equal to the Nyquist frequency, so $\omega=\pi$.

We record the largest power spectrum for all traces [6] in Table 2, showing peak magnitude of daily and weekly frequencies extracted per transatlantic trace (Figure 3). Our aim with this FFT analysis is to study if there is high variability among the traffic traces to justify variance [4].
Table 2: Peak Frequency Capture.

\begin{tabular}{llll}
\hline S/N & Link Traffic & Weekly (dB) & Daily (dB) \\
\hline 1 & Amst_Bost_in & 223.6 & 138.9 \\
2 & Amst_Bost_out & 342.4 & 122.0 \\
3 & Aofa_Lond_in & 92.44 & 143.6 \\
4 & Aofa_Lond_out & 78.95 & 277.1 \\
5 & Wash_Cern_in & 215.4 & 580.5 \\
6 & Wash_Cern_out & 252.0 & 163.5 \\
7 & Lond_NY_in & 260.2 & 145.4 \\
8 & Lond_NY_out & 554.1 & 904.2 \\
\hline
\end{tabular}

\section{STATISTICAL TIME-SERIES MODELS}

ARIMA. ARIMA uses auto-regressive moving averages to predict future points in a time-series model.

ARIMA parameters are selected based on data and their corresponding and partial auto-correlation plots. The tentative models will be fit to the traffic trace, using moving average and autoregressive respectively. In ARIMA, we need to calculate the seasonal differencing and feed these into the model for predictions.

Holt-Winters. These coefficients correspond to respective smoothing equations for level, trend and seasonality where the sequence of observations beginning at time $t=0$ is represented by $x_{t} . s_{t}$ represents the smoothed value of time $t$ and $b_{t}$ represents the best estimate of trend at $t$.

The parameters are tuned using trial-and-error methods, for example, 10 epochs on average.

\section{LSTM-BASED NEURAL NETWORK MODELS}

In ARIMA and Holt-Winters, adding seasonal patterns in advance as model features, can help strengthen simple prediction models [21]. However, this process is error-prone, making it difficult to extract these features in advance. Here, we describe building LSTMbased neural network architectures, which learn features from data during training phase.

\subsection{Problem Formulation and Notation}

We denote $L$ as the number of network links in a WAN network. For each link $l \in L$, traffic is measured as a time-series problem $\left\{y_{(l, t)}\right\}$, where $t \in T$ indicates the hourly time bins and $y$ is the hourly aggregated traffic data (normalized) moving in one direction on the link $l$ in time bin $t$ (Figure 5). 


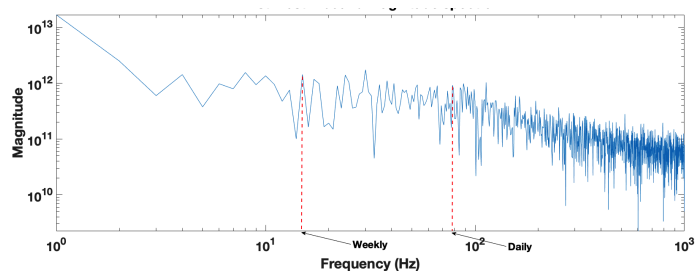

(a) Amst-Bost Inbound.

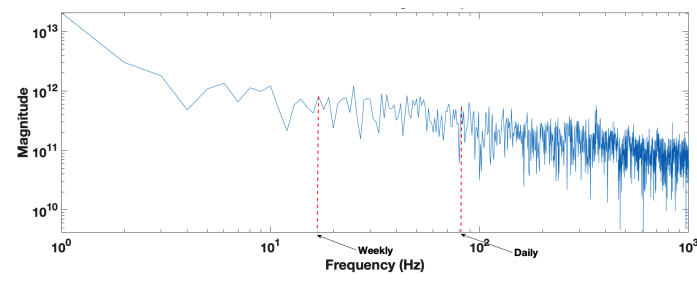

(b) Amst-Bost Outbound.

Figure 3: Extracting frequency magnitude for weekly and daily patterns.

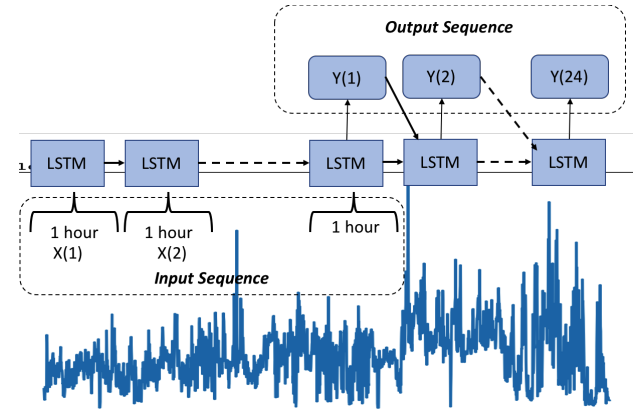

Figure 4: Learning features from the data itself as a sequential problem.
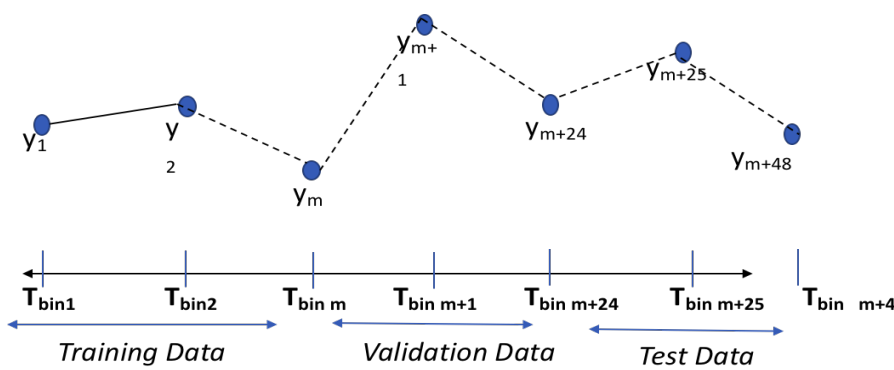

Figure 5: Time bins are 1 hour aggregate traffic. Training, Validation (24 hours) and Test (24 hours).

The training set contains data in time steps $\left[0, t_{m}\right]$, the validation set contains time steps $\left[t_{m+1}, \ldots, t_{m+24}\right]$ using 24 hours to fit the model. The test data is unseen by the model and used to compare as ground truth, $\left[t_{m+25}, \ldots, t_{m+48}\right]$. The prediction output for a link $l$ is defined as $\hat{y}_{(t, l, 0)}, \ldots, \hat{y}_{(t, l, 24)}$, predicting next-24-hours.

5.1.1 Measuring Prediction Accuracy. We use the Root Mean Square Error (RMSE) to evaluate the predicted bandwidth and ground truth as measures of accuracy for the next-24-hours. This is defined as (where $n=24$ ),

$$
R M S E=\sqrt{\left.\frac{1}{n} \sum_{j=1}^{n}\left(y_{(l, j)}-\hat{y}_{(l, j}\right)\right)^{2}}
$$

We chose RMSE because it increases the variance between actual and predicted values, and we want to assess whether error builds up as we predict future values.

\subsection{Model Description}

In this section, we describe the construction of the LSTM designs investigated.

5.2.1 Recurrent Neural Networks. These are a class of neural networks, distinguished from feed-forward networks, that use feedback loops to connect their past decisions to future predictions. Ingesting their own behavior outputs as input [6], allows RNNs to model and perform sequential learning, using the equations:

$$
\begin{gathered}
h_{t}=f_{a c t}\left(W^{(h h)} h_{t-1}+W^{(h x)} x_{t}\right) \\
y_{t}=W^{(S)} h_{t} \\
J^{(t)}(\Theta)=\sum_{i=1}^{|V|}\left(y_{t_{i}} \log \left(y_{t_{i}}\right)\right)
\end{gathered}
$$

Equation 7 defines $h_{t}$ as the current hidden state that contains information about previously observed data, taking inputs from previous hidden states $h_{t-1}$ and current input sequences $x_{t}$. The $8 f_{\text {act }}$ denotes the activation function used in the model. Equation 8 defines $y_{t}$ as the predicted output from the current cell. Equation 9 is the cross-entropy loss function at each time step $t$, that helps train the model by evaluating the error between predicted and actual.

Vanishing gradient problem. RNNs are seen to suffer from the vanishing gradient problem. If we treat each time-step as a separate RNN layer, the neural network becomes very deep, relational to the number of input data points [10]. As we train and repeatedly update the hidden states, gradient updates become smaller and smaller, with the addition of more time-steps. The RNN model thus has trouble learning long-term dependencies i.e. data points at the beginning of the sequence are not remembered as sequence becomes long. This creates a particular problem for learning network traffic data, as it has been proven to contain long-range dependencies [3], making RNNs unsuitable for network traffic.

5.2.2 Long Short-Term Memory Network. To help overcome the vanishing gradient problem, LSTMs use a special memory block units in hidden layer, that store temporal state using gates for allowable information. By controlling information flow at every step, LSTM is able to learn long-term dependencies and maps an input sequence $X=\left(x_{1}, \ldots, x_{T}\right)$ to an output sequence $Y=\left(y_{1}, \ldots, y_{T}\right)$ by using Equations 10-14. 


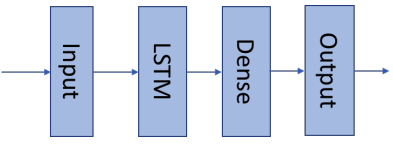

(a) Simple LSTM Model Design.

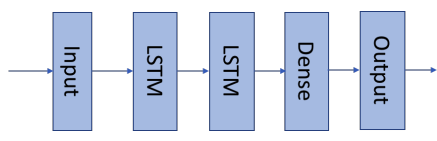

(b) Stacked LSTM Model Design.

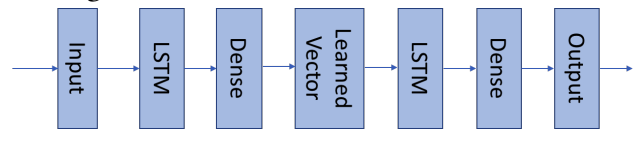

(c) S2S LSTM Model Design.

Figure 6: LSTM-based design variants used in the study.

$$
\begin{gathered}
f_{t}=\sigma\left(W_{f} \cdot\left[h_{t-1}, x_{t}\right]+b_{f}\right) \\
i_{t}=\sigma\left(W_{i} \cdot\left[h_{t-1}, x_{t}\right]+b_{i}\right) \\
h_{t}=o_{t} * \tanh \left(C_{t}\right) \\
\widetilde{C}_{t}=\tanh \left(W_{C} \cdot\left[h_{t-1}, x_{t}\right]+b_{C}\right. \\
C_{t}=f_{t} * C_{t-1}+i_{t} * \widetilde{C}_{t}
\end{gathered}
$$

$f_{t}, i_{t}$, and $\widetilde{C}_{t}$ are the forget gate, input gate, and new candidate values respectively, and $\sigma$ represents activation functions used. The new cell state $C_{t}$ is determined by multiplying the old state $C_{t-1}$ by $f_{t}$ and adds to new candidate values $\widetilde{C}_{t}$ scaled by $i_{t}$, effectively deciding to update each state value. The output $h_{t}$ is determined by considering new cell state with previous output and current input.

\subsection{LSTM architectures}

5.3.1 Simple LSTM Learning. Figure 6a shows the model design for a simple LSTM. The model gathers input data and learns hidden relationships from one input sequence to the next. This way it learns behavior patterns in the data.

Why should this learn good features? To predict the future, the model uses past hourly data to learn how the traffic pattern changes from one input to the next. The hidden state will try to capture this information and extrapolate future hours.

5.3.2 Stacked LSTM Learning. Figure 6b shows the model design stacking two LSTM layers to improve the learned representations. Stacking LSTMs can improve the accuracy of the behavior because it has more LSTM layers. However, this model can also lead to overfitting on the data being studied [8].

Why should this learn good features? It has been seen that adding hidden layers can improve the learned sparse behavior in the data, improving the accuracy obtained [9]. This design also has a larger size neural network and would require more computational time to train.

5.3.3 Sequence-to-Sequence (S2S) Learning. Figure 6c shows S2S learning method has two main components, an encoder and a decoder layer. The encoder LSTM layer learns behavior representation from the input sequence and generates a fixed-size context vector, also known as the 'learned vector'. This context vector captures features and uses these to project the decoder output, also represented by another LSTM layer. The context vector can be used to project future data behaviors.
Why should this learn good features? Traditionally, the S2S LSTM (autoencoder) design has worked well for language translation but is not generalizable to many-many problems [17]. In our experiment, we learn the context vector by passing training data between the encoder-decoder sequences, learning finer-grained behavior. This architecture allows S2S LSTM to learn and remember information for longer time periods, practical for multiple steps.

\section{EXPERIMENT AND RESULTS}

We implemented ARIMA, Holt-Winters and three LSTM variants in Python, using Keras and TensorFlow as the backend. The next24-hour predictions are compared with ground truth values on all 8 traffic traces.

ARIMA and Holt-Winters. To select parameters for the ARIMA model, we inspected the traffic data plots and their corresponding autocorrelation (AC) and partial autocorrelation (PAC) plots. These plots indicated that the series for each of the selected link traces is stationary, so the model did not require differencing. Furthermore, the plots also indicate that the series is not over-differenced, such the model does not require an MA term. To account for the possibility of under-differencing, we added one AR term (i.e. $p=1$ ) to the model. The Holt-Winters model coefficients correspond to the respective smoothing equations. Tuned using trial-and-error for 10 epochs, we settled on coefficients of $0.7,0.02,0.9$ for level, trend and seasonality respectively.

LSTM models and Hyperparameter tuning. For the next-24hour prediction, the dimension of LSTM outputs is set to output = 24 . We train the LSTM on the available days' data $(t=0, \ldots, n-1)$ and use the last day $n$ as ground truth. When building the LSTM models, we tested the model with 10, 24 and 50 nodes in hidden layers, but found that the performance did not change much.Therefore, we use 24 nodes as the standard across all the models in the comparison. For LSTM training, we use the Adam optimizer [16] (with default parameters e.g. learning rate, beta, etc.). We tested the activation functions and dropouts.

Each model was trained for 10 epochs and run 3 times each, to average results. The variance is shown using error bars in the 24-hour predictions.

Training, Validation and Testing for Transatlantic links. We used 90 days of transatlantic traffic data. We use the first 88 days (2112 hours) as training data, the $89^{\text {th }}$ day ( 24 hours) as validation data to fit the model, and the $90^{t h}$ day (24 hours) as unseen test or ground truth data. Predictions are done using the $89^{t h}$ day as input. 
6.0.1 Results. The complete prediction result for all the models is presented in Table 3. Figure 9 shows the corresponding next-24hour predictions with all 5 models.

Overall, the LSTM models show considerably low RMSE across all transatlantic links except Aofa-London-Inbound and Wash-CernInbound. The negative percentages show that Holt-Winters performs better in these with $-67 \%$ and $-163 \%$ respectively. Aofa-LondonInbound (Figure 9c) show that the LSTM models and Holt-Winters are all anticipating a high traffic trend, but ground truth traffic is much lower. Holt-Winters was able to anticipate this lower trend.

The Wash-Cern-Inbound also performs badly, anticipating much higher traffic trends. Observing the original data trace, Figure2d shows that there is downtime for 2 weeks in November, where the device did not record data, the training data was faulty.

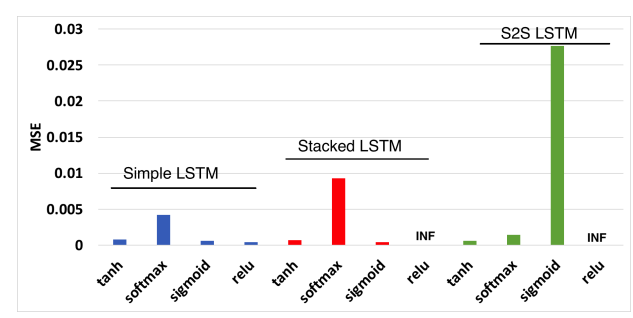

Figure 7: Multiple activation functions on AmsterdamBoston-Outbound.

The early predictions show some movement but taper off into a trend line. Lacking the valleys and peaks, the LSTM models fail to remember the peak behaviors for further steps. In all 8 cases, the trends are higher than the actual bandwidth. Having more training data and using more layers can help the model remember behaviors.

Varying the activation functions. Figure 7 shows the Mean Square Error (MSE) $\left(R M S E^{2}\right)$ for the link Amsterdam-Boston-Outbound. The graph shows that tanh performs the best across all LSTM design variants. The activation function relu results in 'INF' in Stacked and S2S LSTM. This is because the loss starts to grow significantly from epoch to epoch, eventually becoming too large to be represented as a floating point. This condition is also known as gradient blow up.

Adding dropout in S2S LSTM. Dropout prevents over-fitting in the LSTM layers, by making the training process noisy, forcing nodes to use a probability to remember the inputs. It make improve the generalizability of the model [15]. Table 4 shows that in some cases Aofa-London-Inbound, Wash-Cern-Inbound and LondNY-Outbound, adding a Dropout (0.25) significantly improved the RMSE. In the Aofa-London-Inbound case, adding the dropout performs better than the Holt-Winters predictions. Therefore this can be a useful way of making the deep learning models more robust.

Computational Overhead: To validate the feasibility of offline training and online prediction, we assessed the computational overhead of the LSTM models. Testing on our CPU (Intel Core i7 $(2.5 \mathrm{GHz}$ $1600 \mathrm{MHz} 2048 \mathrm{MB})$. Shown in Figure 8, S2S LSTM takes the longest time to train and predict, with no gain on the RMSE. As such, using a Simple or Stacked LSTM will be more suitable to use.

Deploying Deep Learning in Practice. Our results show that data-driven unsupervised learning can aid in better predictions

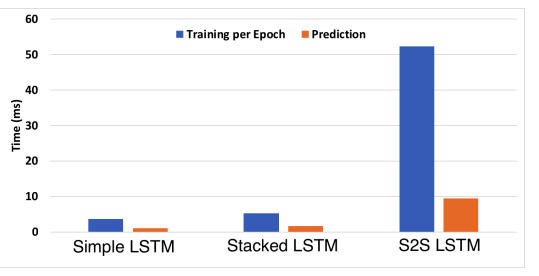

Figure 8: Computational Time.

compared to statistical methods. No global parameters have to be set in advance that consider seasonality.

Our Limitations. First, we chose only links with long TCP return times such as transatlantic. These links have a usual capacity of $100 \mathrm{~GB} / \mathrm{s}-400 \mathrm{~GB} / \mathrm{s}$ and have large traffic volumes. Checking whether our techniques work on smaller capacity links (of 10GB) can introduce more random spikes that may lead to bottlenecks.

\section{RELATED WORK}

To the best of our knowledge, no prior work has compared LSTMvariants in real network traffic traces. When developing this solution, we were inspired by two areas of work.

Network Traffic Prediction. Seminal works of Wolski et al. [20] highlighted the importance of network forecasting to improve TCP performance for scheduling computations. Traditional time-series prediction methods such as ARIMA, Holt-Winters, BoxJenkins and Hidden Markov Models, all model time data as stochastic processes. These are successful in predicting a few time-steps ahead [2], but the accuracy depends on data exhibiting seasonality. Network spikes are difficult to anticipate and methods deviate significantly on volatile real statistics [18]. Statistical techniques also fail to learn long-range dependency [12].

The random nature of network traffic makes it difficult to predict [21]. [3] trained LSTMs on GEANT network to predict 15 minutes.

Fourier transform and machine learning. FT can describe dynamic responses and identify regular contributions to fluctuating signals, exploring patterns in the frequency domain [4]. FFT has also been used in traffic flow to model non-linear spatiotemporal effects and there is potential to combine it with deep learning approaches [13]. In this study, we used FFT to perform seasonality analysis to detect strong peak frequency pattern and amplitude in the data just to show variability.

\section{CONCLUSIONS}

Our paper findings have a number of implications for network prediction research and engineering community. Our evaluation yields important decisions for deploying prediction tools with traffic monitoring tools and can have an immediate impact on network utilization. Currently, our methods are being optimized for next-24hour prediction only, there is potential for further research to extend the prediction periods. Additionally, experimenting with advanced neural network architectures can help improve the accuracy. 


\begin{tabular}{|l|l|l|l|l|l|l|l|l|}
\hline Model & Ams-Bst-O & Ams-Bst-I & Af-Lnd-O & Af-Lnd-I & Wsh-Crn-O & Wsh-Crn-I & Lnd-NY-O & Lnd-NY-I \\
\hline ARIMA & 0.1630 & 0.1142 & 0.1575 & 0.1244 & 0.0418 & 0.0538 & 0.1087 & 0.1212 \\
\hline Holt-Winters & 0.2172 & 0.3624 & 0.2473 & $\mathbf{0 . 0 6 2 6}$ & 0.0340 & $\mathbf{0 . 0 4 3 9}$ & 0.1098 & 0.1052 \\
\hline Simple LSTM & 0.0502 & 0.0771 & 0.0791 & 0.1048 & $\mathbf{0 . 0 2 6 8}$ & 0.1156 & $\mathbf{0 . 0 5 5 7}$ & 0.0320 \\
\hline Stacked LSTM & $\mathbf{0 . 0 4 0 7}$ & $\mathbf{0 . 0 7 6 4}$ & $\mathbf{0 . 0 6 6 0}$ & 0.0992 & 0.0290 & 0.1129 & 0.0885 & $\mathbf{0 . 0 2 9 3}$ \\
\hline S2S LSTM & 0.0531 & 0.0801 & 0.0814 & 0.0949 & 0.0307 & 0.1802 & 0.0580 & 0.0293 \\
\hline \hline \multicolumn{7}{|l|}{ Summary: LSTMs better than ARIMA [AR] and HW (\%) } \\
\hline SimpleLSTM over AR & $69 \%$ & $33 \%$ & $50 \%$ & $16 \%$ & $36 \%$ & $-114 \%$ & $49 \%$ & $74 \%$ \\
\hline StackLSTM over AR & $75 \%$ & $33 \%$ & $58 \%$ & $20 \%$ & $31 \%$ & $-109 \%$ & $19 \%$ & $76 \%$ \\
\hline S2S LSTM over AR & $67 \%$ & $30 \%$ & $48 \%$ & $23 \%$ & $26 \%$ & $-234 \%$ & $47 \%$ & $76 \%$ \\
\hline \hline SimpleLSTM over HW & $76 \%$ & $79 \%$ & $68 \%$ & $-67 \%$ & $21 \%$ & $-163 \%$ & $49 \%$ & $70 \%$ \\
\hline StackLSTM over HW & $81 \%$ & $79 \%$ & $73 \%$ & $-58 \%$ & $15 \%$ & $-157 \%$ & $19 \%$ & $73 \%$ \\
\hline S2S LSTM over HW & $76 \%$ & $78 \%$ & $67 \%$ & $-51 \%$ & $10 \%$ & $-310 \%$ & $47 \%$ & $72 \%$ \\
\hline
\end{tabular}

Table 3: RMSE of predicted next-24-hours Transatlantic links.

\begin{tabular}{|l|l|l|l|l|l|l|l|l|}
\hline Model & Ams-Bst-O & Ams-Bst-I & Af-Lnd-O & Af-Lnd-I & Wsh-Crn-O & Wsh-Crn-I & Lnd-NY-O & Lnd-NY-I \\
\hline No dropout & $\mathbf{0 . 0 5 3 1}$ & $\mathbf{0 . 0 8 0 1}$ & $\mathbf{0 . 0 8 1 4}$ & 0.0949 & $\mathbf{0 . 0 3 0 7}$ & 0.1802 & 0.0580 & $\mathbf{0 . 0 2 9 3}$ \\
\hline Dropout $=0.25$ & 0.1647 & 0.0955 & 0.0878 & $\mathbf{0 . 0 3 9 4}$ & 0.0518 & $\mathbf{0 . 0 9 8 2}$ & $\mathbf{0 . 0 5 5 2}$ & 0.0467 \\
\hline Dropout $=0.4$ & 0.1650 & 0.1157 & 0.0882 & 0.0402 & 0.0484 & 0.2230 & 0.0556 & 0.0872 \\
\hline
\end{tabular}

Table 4: Adding Dropout 0.25, 0.4 to S2S LSTM.

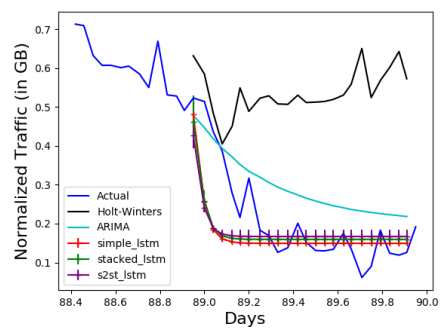

(a) Amsterdam-Boston Inbound.

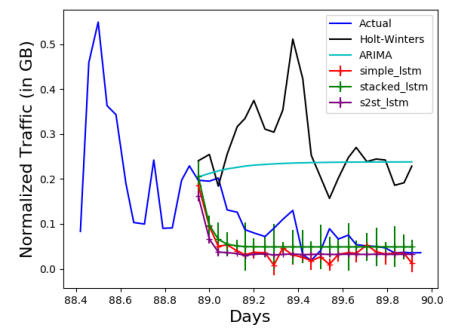

(b) Amsterdam-Boston Outbound.

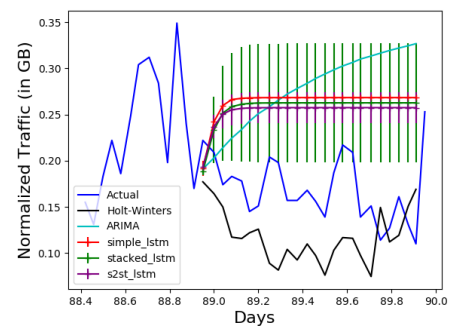

(c) Aofa-London Inbound.

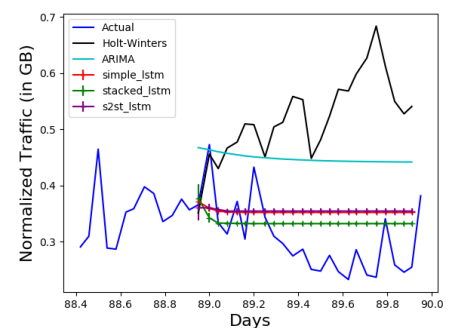

(d) Aofa-London Outbound.

Figure 9: Next-24-hour prediction for Transatlantic Traffic.

\section{ACKNOWLEDGEMENTS}

This work was supported by the U.S. Department of Energy, Office of Science Early Career Research Program, under LAB 16-1625 for Deep Learning FP00006145.

\section{REFERENCES}

[1] Ghazi Al-Naymat, Mouhammd Al-kasassbeh, and Eshraq Al-Hawari. 2018. Exploiting SNMP-MIB Data to Detect Network Anomalies using Machine Learning Techniques. In SAI Intelligent Systems Conf. 991-1004.

[2] J. Scott Armstrong. 2001. Principles of Forecasting: A Handbook for Researchers and Practitioners. Kluwer Academic Publishers (2001).

[3] Abdelhadi Azzouni and Guy Pujolle. 2018. NeuTM: A neural network-based framework for traffic matrix prediction in SDN. NOMS (2018).

[4] Peter Bloomfield. 2004. Fourier analysis of time series: an introduction. John Wiley \& Sons.

[5] Cisco. 2019. Trending analysis. https://www.cisco.com/c/en/us/solutions/ collateral/service-provider/visual-networking-index-vni/vni-hyperconnectivitywp.html (2019).

[6] J. T. Connor, R. D. Martin, and L. E. Atlas. 1994. Recurrent Neural Networks and Robust Time Series Prediction. Trans. Neur. Netw. 5, 2 (March 1994), 240-254.

[7] P. Cortez, M. Rio, M. Rocha, and P. Sousa. 2006. Internet Traffic Forecasting using Neural Networks. IEEE Int. foint Conf. Neural Network Proceedings (2006).
[8] Aaron C. Courville, Ian Goodfellow, and Yoshua Bengio. 2015. Deep Learning. (2015)

[9] G.E. Hinton. 2007. Learning multiple layers of representation. Trends in Cognitive Science (2007), 428-434.

[10] Sepp Hochreiter and Jürgen Schmidhuber. 1997. Long Short-Term Memory. Neural Comput. 9, 8 (Nov. 1997), 1735-1780.

[11] Sushant Jain, Alok Kumar, Subhasree Mandal, Joon Ong, Leon Poutievski, Arjun Singh, Subbaiah Venkata, Jim Wanderer, Junlan Zhou, Min Zhu, Jon Zolla, Urs Hölzle, Stephen Stuart, and Amin Vahdat. 2013. B4: Experience with a Globallydeployed Software Wan. SIGCOMM Comput. Commun. Rev. 43, 4 (2013), 3-14.

[12] Mbulelo Brenwen Ntlangu and Alireza Baghai-Wadji. 2017. Modeling Network Traffic Using Time Series Analysis: A Review. In Int. Conf. Big Data and IoT. 209-215.

[13] Nicholas G Polson and Vadim O Sokolov. 2017. Deep learning for short-term traffic flow prediction. Trans. Res. Part C: Emerging Tech. 79 (2017), 1-17.

[14] Kim Roberts, Qunbi Zhuge, Inder Monga, Sebastien Gareau, and Charles Laperle. 2017. Beyond $100 \mathrm{~Gb} / \mathrm{s}$ : capacity, flexibility, and network optimization. Fournal Optical Comm. and Networking 9, 4 (2017).

[15] Nitish Srivastava, Geoffrey Hinton, Alex Krizhevsky, Ilya Sutskever, and Ruslan Salakhutdinov. 2014. Dropout: A Simple Way to Prevent Neural Networks from Overfitting. F. Mach. Learn. Res. 15, 1 (2014), 1929-1958.

[16] Nitish Srivastava, Elman Mansimov, and Ruslan Salakhutdinov. 2015. Unsupervised Learning of Video Rep. Using LSTMs. In ICML. 843-852. 
[17] Ilya Sutskever, Oriol Vinyals, and Quoc V Le. 2014. Sequence to Sequence Learning with Neural Networks. In NIPS. 3104-3112.

[18] Hao Wang, Haiyong Xie, Lili Qiu, Yang Richard Yang, Yin Zhang, and Albert Greenberg. [n.d.]. COPE: Traffic Engineering in Dynamic Networks. SIGCOMM Comp. Comm. 36, 4 ([n. d.]), 99-110.

[19] Weitao Wang, Yuebin Bai, Chao Yu, Yuhao Gu, Peng Feng, Xiaojing Wang, and Rui Wang. 2018. A network traffic flow prediction with deep learning approach for large-scale metropolitan area network. NOMS (2018).

[20] Rich Wolski, Neil T. Spring, and Jim Hayes. 1999. The Network Weather Service: A Distributed Resource Performance Forecasting Service for Metacomputing. Future Gener. Comput. Syst. 15, 5-6 (Oct. 1999), 757-768.

[21] Wucherl Yoo and Alex Sim. 2016. Time-Series Forecast Modeling on HighBandwidth Network Measurements. f. Grid Comput. 14, 3 (Sept. 2016), 463-476. 